Bull. Korean Math. Soc. 48 (2011), No. 4, pp. 853-871

DOI 10.4134/BKMS.2011.48.4.853

\title{
STABILITY OF AN ADDITIVE FUNCTIONAL INEQUALITY IN PROPER $C Q^{*}$-ALGEBRAS
}

\author{
Jung Rye Lee, Choonkil Park, And Dong Yun Shin
}

ABstract. In this paper, we prove the Hyers-Ulam-Rassias stability of the following additive functional inequality:

$(0.1) \quad\|f(2 x)+f(2 y)+2 f(z)\| \leq\|2 f(x+y+z)\|$.

We investigate homomorphisms in proper $C Q^{*}$-algebras and derivations on proper $C Q^{*}$-algebras associated with the additive functional inequality $(0.1)$.

\section{Introduction and preliminaries}

Ulam [36] gave a talk before the Mathematics Club of the University of Wisconsin in which he discussed a number of unsolved problems. Among these was the following question concerning the stability of homomorphisms.

We are given a group $G$ and a metric group $G^{\prime}$ with metric $\rho(\cdot, \cdot)$. Given $\epsilon>$ 0 , does there exist a $\delta>0$ such that if $f: G \rightarrow G^{\prime}$ satisfies $\rho(f(x y), f(x) f(y))<$ $\delta$ for all $x, y \in G$, then a homomorphism $h: G \rightarrow G^{\prime}$ exists with $\rho(f(x), h(x))<$ $\epsilon$ for all $x \in G$ ?

By now an affirmative answer has been given in several cases, and some interesting variations of the problem have also been investigated.

Hyers [13] considered the case of approximately additive mappings $f: E \rightarrow$ $E^{\prime}$, where $E$ and $E^{\prime}$ are Banach spaces and $f$ satisfies Hyers inequality

$$
\|f(x+y)-f(x)-f(y)\| \leq \epsilon
$$

for all $x, y \in E$. It was shown that the limit

$$
L(x)=\lim _{n \rightarrow \infty} \frac{f\left(2^{n} x\right)}{2^{n}}
$$

exists for all $x \in E$ and that $L: E \rightarrow E^{\prime}$ is the unique additive mapping satisfying

$$
\|f(x)-L(x)\| \leq \epsilon .
$$

Received January 18, 2010; Revised March 13, 2010.

2010 Mathematics Subject Classification. Primary 39B72; Secondary 47N50, 47L60, 46H35, 47Jxx, 47L90, 46B03.

Key words and phrases. additive functional inequality, Hyers-Ulam-Rassias stability, proper $C Q^{*}$-algebras, proper $C Q^{*}$-algebra homomorphism, derivation. 
Th. M. Rassias [25] provided a generalization of Hyers' Theorem which allows the Cauchy difference to be unbounded.

Theorem 1.1 (Th. M. Rassias). Let $f: E \rightarrow E^{\prime}$ be a mapping from a normed vector space $E$ into a Banach space $E^{\prime}$ subject to the inequality

$$
\|f(x+y)-f(x)-f(y)\| \leq \theta\left(\|x\|^{p}+\|y\|^{p}\right)
$$

for all $x, y \in E$, where $\theta$ and $p$ are positive real numbers with $p<1$. Then the limit

$$
L(x)=\lim _{n \rightarrow \infty} \frac{f\left(2^{n} x\right)}{2^{n}}
$$

exists for all $x \in E$ and $L: E \rightarrow E^{\prime}$ is the unique additive mapping which satisfies

$$
\|f(x)-L(x)\| \leq \frac{2 \theta}{2-2^{p}}\|x\|^{p}
$$

for all $x \in E$. Also, if for each $x \in E$ the function $f(t x)$ is continuous in $t \in \mathbb{R}$, then $L$ is $\mathbb{R}$-linear.

Th. M. Rassias [26] during the $27^{\text {th }}$ International Symposium on Functional Equations asked the question whether such a theorem can also be proved for $p \geq 1$. Gajda [11] following the same approach as in Th. M. Rassias [25], gave an affirmative solution to this question for $p>1$. It was shown by Gajda [11], as well as by Th. M. Rassias and Šemrl [31] that one cannot prove a Th. M. Rassias' type theorem when $p=1$. The counterexamples of Gajda [11], as well as of Th. M. Rassias and Šemrl [31] have stimulated several mathematicians to invent new definitions of approximately additive or approximately linear mappings, cf. P. Găvruta [12], who among others studied the Hyers-Ulam-Rassias stability of functional equations. The inequality (1.1) that was introduced for the first time by Th. M. Rassias [25] provided a lot of influence in the development of a generalization of the Hyers-Ulam stability concept. This new concept is known as Hyers-Ulam-Rassias stability of functional equations (cf. the books of P. Czerwik [9, 10], D. H. Hyers, G. Isac and Th. M. Rassias [14]).

Beginning around the year 1980, the topic of approximate homomorphisms and their stability theory in the field of functional equations and inequalities was taken up by several mathematicians (cf. D. H. Hyers and Th. M. Rassias [15], Th. M. Rassias [29] and the references therein).

J. M. Rassias [23] following the spirit of the innovative approach of Th. M. Rassias [25] for the unbounded Cauchy difference proved a similar stability theorem in which he replaced the factor $\|x\|^{p}+\|y\|^{p}$ by $\|x\|^{p} \cdot\|y\|^{q}$ for $p, q \in \mathbb{R}$ with $p+q \neq 1$ (see also [24] for a number of other new results).

Theorem 1.2 ([22, 23, 24]). Let $X$ be a real normed linear space and $Y$ a real complete normed linear space. Assume that $f: X \rightarrow Y$ is an approximately additive mapping for which there exist constants $\theta \geq 0$ and $p \in \mathbb{R}-\{1\}$ such 
that $f$ satisfies inequality

$$
\|f(x+y)-f(x)-f(y)\| \leq \theta \cdot\|x\|^{\frac{p}{2}} \cdot\|y\|^{\frac{p}{2}}
$$

for all $x, y \in X$. Then there exists a unique additive mapping $L: X \rightarrow Y$ satisfying

$$
\|f(x)-L(x)\| \leq \frac{\theta}{\left|2^{p}-2\right|}\|x\|^{p}
$$

for all $x \in X$. If, in addition, $f: X \rightarrow Y$ is a mapping such that the transformation $t \rightarrow f(t x)$ is continuous in $t \in \mathbb{R}$ for each fixed $x \in X$, then $L$ is an $\mathbb{R}$-linear mapping.

Several mathematicians have contributed to this subject (see [16]-[20], [27][30], [32]).

In this paper, we prove the Hyers-Ulam-Rassias stability of the following additive functional equation:

$$
\|f(2 x)+f(2 y)+2 f(z)\| \leq\|2 f(x+y+z)\| .
$$

In a series of papers [1]-[8] and [33]-[35], many authors have considered a special class of quasi *-algebras, called proper $C Q^{*}$-algebras, which arise as completions of $C^{*}$-algebras. They can be introduced in the following way:

Let $A$ be a Banach module over the $C^{*}$-algebra $A_{0}$ with involution $*$ and $C^{*}$-norm $\|\cdot\|_{0}$ such that $A_{0} \subset A$. We say that $\left(A, A_{0}\right)$ is a proper $C Q^{*}$-algebra if

(i) $A_{0}$ is dense in $A$ with respect to its norm $\|\cdot\|$;

(ii) an involution $*$, which extends the involution of $A_{0}$, is defined in $A$ with the property $(x y)^{*}=y^{*} x^{*}$ for all $x, y \in A$ whenever multiplications $x y$ and $y^{*} x^{*}$ are defined;

(iii) $\|y\|_{0}=\sup _{x \in A,\|x\| \leq 1}\|x y\|$ for all $y \in A_{0}$.

Definition 1.3. Let $\left(A, A_{0}\right)$ and $\left(B, B_{0}\right)$ be proper $C Q^{*}$-algebras.

(i) A $\mathbb{C}$-linear mapping $h: A \rightarrow B$ is called a proper $C Q^{*}$-algebra homomorphism if $h(x y)=h(x) h(y)$ for all $x, y \in A$ whenever multiplications $x y$ and $h(x) h(y)$ are defined. In this case, we say that $h(x y)=h(x) h(y)$ for all $x, y \in A$ whenever the multiplication is defined.

(ii) A $\mathbb{C}$-linear mapping $\delta: A \rightarrow A$ is called a derivation on $A$ if $\delta(x y)=$ $\delta(x) y+x \delta(y)$ for all $x, y \in A$ whenever multiplications $x y, \delta(x) y$, and $x \delta(y)$ are defined. In this case we say $\delta(x y)=\delta(x) y+x \delta(y)$ for all $x, y \in A$ whenever the multiplication is defined.

The purpose of this paper is to investigate the stability of homomorphisms in proper $C Q^{*}$-algebras and of derivations on proper $C Q^{*}$-algebras associated with the additive functional inequality (0.1). In this paper, we use only the property that a $C Q^{*}$-algebra is a Banach space with a partially defined multiplication. Therefore our results are generalized to a Banach space with a partially defined multiplication. 
This paper is organized as follows: In Section 2, we prove the stability of $\mathbb{C}$-linear mappings in Banach spaces.

In Section 3, we prove the stability of homomorphisms in proper $C Q^{*}$ algebras.

In Section 4, we prove the stability of derivations on proper $C Q^{*}$-algebras.

Throughout this paper, we assume that $\left(A, A_{0}\right)$ is a proper $C Q^{*}$-algebra with $C^{*}$-norm $\|\cdot\|_{A_{0}}$ and norm $\|\cdot\|_{A}$, and that $\left(B, B_{0}\right)$ is a proper $C Q^{*}$-algebra with $C^{*}$-norm $\|\cdot\|_{B_{0}}$ and norm $\|\cdot\|_{B}$. We denote that $\mathbb{T}^{1}:=\{\lambda \in \mathbb{C}|| \lambda \mid=1\}$.

\section{Stability of $\mathbb{C}$-linear mappings in Banach spaces}

We investigate the Hyers-Ulam-Rassias stability of $\mathbb{C}$-linear mappings in Banach spaces associated with the additive functional inequality. In this section, we assume that $X, Y$ are Banach spaces.

Lemma 2.1. Let $f: X \rightarrow Y$ be a mapping satisfying

$$
\|f(2 x)+f(2 y)+2 f(z)\|_{Y} \leq\|2 f(x+y+z)\|_{Y}
$$

for all $x, y, z \in X$. Then $f$ is additive.

Proof. Letting $x=y=z=0$ in (2.1), we get $\|4 f(0)\|_{Y} \leq\|2 f(0)\|_{Y}$ and so $f(0)=0$.

Letting $z=0$ and replacing $y$ by $-x$ in (2.1), we get

$$
\|f(2 x)+f(-2 x)\|_{Y} \leq\|2 f(0)\|_{Y}=0
$$

for all $x \in X$. Hence $f(-2 x)=-f(2 x)$ and so $f(-x)=-f(x)$ for all $x \in X$.

Letting $y=0$ and replacing $z$ by $-x$ in (2.1), we get

$$
\|f(2 x)+2 f(-x)\|_{Y} \leq\|2 f(0)\|_{Y}=0
$$

for all $x \in X$. Thus we have $f(2 x)=2 f(x)$ for all $x \in X$.

Letting replacing $z$ by $-x-y$ in (2.1), we get

$$
\|f(2 x)+f(2 y)-2 f(x+y)\|_{Y} \leq\|2 f(0)\|_{Y}=0
$$

for all $x, y \in X$. Thus we have

$$
f(2 x+2 y)=f(2 x)+f(2 y)
$$

and so

for all $x, y \in X$, as desired.

$$
f(x+y)=f(x)+f(y)
$$

Theorem 2.2. Let $f: X \rightarrow Y$ be a mapping with $f(0)=0$. If there exists a function $\varphi: X^{3} \rightarrow[0, \infty)$ satisfying

$$
\begin{aligned}
& \|f(2 x)+f(2 y)+2 f(z)\|_{Y} \leq\|2 f(x+y+z)\|_{Y}+\varphi(x, y, z), \\
& \widetilde{\varphi}(x, y, z):=\sum_{j=0}^{\infty} \frac{1}{2^{j}} \varphi\left((-2)^{j} x,(-2)^{j} y,(-2)^{j} z\right)<\infty
\end{aligned}
$$


for all $x, y, z \in X$, then there exists a unique additive mapping $L: X \rightarrow Y$ satisfying

$$
\|f(x)-L(x)\|_{Y} \leq \frac{1}{2} \widetilde{\varphi}(0,-x, x)
$$

for all $x \in X$.

Proof. Replacing $x, y, z$ by $0,-(-2)^{n} x,(-2)^{n} x$, respectively, and dividing by $2^{n+1}$ in $(2.2)$, since $f(0)=0$, we get

$$
\left\|\frac{f\left((-2)^{n+1} x\right)}{(-2)^{n+1}}-\frac{f\left((-2)^{n} x\right)}{(-2)^{n}}\right\|_{Y} \leq \frac{1}{2^{n+1}} \varphi\left(0,-(-2)^{n} x,(-2)^{n} x\right)
$$

for all $x \in X$. From the above inequality, we have

$$
\begin{aligned}
\left\|\frac{f\left((-2)^{n} x\right)}{(-2)^{n}}-\frac{f\left((-2)^{q} x\right)}{(-2)^{q}}\right\|_{Y} & \leq \sum_{j=q}^{n-1}\left\|\frac{f\left((-2)^{j+1} x\right)}{(-2)^{j+1}}-\frac{f\left((-2)^{j} x\right)}{(-2)^{j}}\right\|_{Y} \\
& \leq \sum_{j=q}^{n-1} \frac{1}{2^{j+1}} \varphi\left(0,-(-2)^{j} x,(-2)^{j} x\right)
\end{aligned}
$$

for all $x \in X$ and all non negative integers $q, n$ with $q<n$. From (2.3), the sequence $\left\{\frac{f\left((-2)^{n} x\right)}{(-2)^{n}}\right\}$ is a Cauchy sequence for all $x \in X$. Since $Y$ is complete, the sequence $\left\{\frac{f\left((-2)^{n} x\right)}{(-2)^{n}}\right\}$ converges for all $x \in X$. So we can define a mapping $L: X \rightarrow Y$ by

$$
L(x):=\lim _{n \rightarrow \infty} \frac{f\left((-2)^{n} x\right)}{(-2)^{n}}
$$

for all $x \in X$.

In order to prove that $L$ satisfies (2.4), if we put $q=0$ and let $n \rightarrow \infty$ in the above inequality then we obtain

$$
\|f(x)-L(x)\|_{Y} \leq \sum_{j=0}^{\infty} \frac{1}{2^{j+1}} \varphi\left(0,-(-2)^{j} x,(-2)^{j} x\right)=\frac{1}{2} \widetilde{\varphi}(0,-x, x)
$$

for all $x \in X$.

Replacing $x, y, z$ by $(-2)^{n} x,(-2)^{n} y,(-2)^{n} z$, respectively, and dividing by $2^{n}$ in (2.2), we get

$$
\begin{aligned}
& \left\|\frac{f\left((-2)^{n} 2 x\right)}{(-2)^{n}}+\frac{f\left((-2)^{n} 2 y\right)}{(-2)^{n}}+\frac{2 f\left((-2)^{n} z\right)}{(-2)^{n}}\right\|_{Y} \\
\leq & \left\|2 \frac{f\left((-2)^{n}(x+y+z)\right)}{(-2)^{n}}\right\|_{Y}+\frac{1}{2^{n}} \varphi\left((-2)^{n} x,(-2)^{n} y,(-2)^{n} z\right)
\end{aligned}
$$

for all $x, y, z \in X$. Since (2.3) gives that

$$
\lim _{n \rightarrow \infty} \frac{1}{2^{n}} \varphi\left((-2)^{n} x,(-2)^{n} y,(-2)^{n} z\right)=0
$$


for all $x, y, z \in X$, if we let $n \rightarrow \infty$ in the above inequality, then we get

$$
\|L(2 x)+L(2 y)+2 L(z)\|_{Y} \leq\|2 L(x+y+z)\|_{Y},
$$

and so $L$ is additive by Lemma 2.1 .

Now to prove the uniqueness of $L$, let $L^{\prime}: X \rightarrow Y$ be another additive mapping satisfying (2.4). Since both $L$ and $L^{\prime}$ are additive, we have

$$
\begin{aligned}
\left\|L(x)-L^{\prime}(x)\right\|_{Y} & =\frac{1}{2^{n}}\left\|L\left(2^{n} x\right)-L^{\prime}\left(2^{n} x\right)\right\|_{Y} \\
& \leq \frac{1}{2^{n}}\left(\left\|L\left(2^{n} x\right)-f\left(2^{n} x\right)\right\|_{Y}+\left\|L^{\prime}\left(2^{n} x\right)-f\left(2^{n} x\right)\right\|_{Y}\right) \\
& \leq \frac{1}{2^{n}} \cdot 2 \widetilde{\varphi}\left(0,-2^{n} x, 2^{n} x\right) \\
& =2 \sum_{j=0}^{\infty} \frac{1}{2^{n+j}} \varphi\left(0,(-2)^{j+n} x,(-2)^{j+n} x\right)
\end{aligned}
$$

which goes to zero as $n \rightarrow \infty$ for all $x \in X$ by (2.3). Consequently, $L$ is a unique additive mapping satisfying (2.4), as desired.

Corollary 2.3. Let $f: X \rightarrow Y$ be a mapping. If there exists a function $\varphi: X^{3} \rightarrow[0, \infty)$ satisfying $(2.2)$ and

$$
\widetilde{\varphi}(x, y, z):=\sum_{i=1}^{\infty} 2^{i} \varphi\left(\frac{x}{(-2)^{i}}, \frac{y}{(-2)^{i}}, \frac{z}{(-2)^{i}}\right)<\infty
$$

for all $x, y, z \in X$, then there exists a unique additive mapping $L: X \rightarrow Y$ satisfying

$$
\|f(x)-L(x)\|_{Y} \leq \frac{1}{2} \widetilde{\varphi}(0,-x, x)
$$

for all $x \in X$.

Proof. Since $\widetilde{\varphi}(0,0,0)<\infty$ in $(2.5)$, we have $\varphi(0,0,0)=0$ and so $f(0)=0$. Replacing $x, y, z$ by $0,-\frac{x}{(-2)^{n}}, \frac{x}{(-2)^{n}}$, respectively, and multiplying by $2^{n-1}$ in (2.2), we get

$$
\left\|(-2)^{n-1} f\left(\frac{x}{(-2)^{n-1}}\right)-(-2)^{n} f\left(\frac{x}{(-2)^{n}}\right)\right\|_{Y} \leq 2^{n-1} \varphi\left(0,-\frac{x}{(-2)^{n}}, \frac{x}{(-2)^{n}}\right)
$$

for all $x \in X$. From the above inequality, we have

$$
\begin{aligned}
& \left\|(-2)^{n} f\left(\frac{x}{(-2)^{n}}\right)-(-2)^{q} f\left(\frac{x}{(-2)^{q}}\right)\right\|_{Y} \\
\leq & \sum_{i=q+1}^{n}\left\|(-2)^{i} f\left(\frac{x}{(-2)^{i}}\right)-(-2)^{i-1} f\left(\frac{x}{(-2)^{i-1}}\right)\right\|_{Y} \\
\leq & \sum_{i=q+1}^{n} 2^{i-1} \varphi\left(0,-\frac{x}{(-2)^{i}}, \frac{x}{(-2)^{i}}\right)
\end{aligned}
$$


for all $x \in X$ and all non negative integers $q, n$ with $q<n$. From (2.5), the sequence $\left\{(-2)^{n} f\left(\frac{x}{(-2)^{n}}\right)\right\}$ is a Cauchy sequence for all $x \in X$. Since $Y$ is complete, the sequence $\left\{(-2)^{n} f\left(\frac{x}{(-2)^{n}}\right)\right\}$ converges for all $x \in X$. So we can define a mapping $L: X \rightarrow Y$ by

$$
L(x):=\lim _{n \rightarrow \infty}(-2)^{n} f\left(\frac{x}{(-2)^{n}}\right)
$$

for all $x \in X$.

In order to prove that $L$ satisfies (2.6), if we put $q=0$ and let $n \rightarrow \infty$ in the above inequality, then we obtain

$$
\|f(x)-L(x)\|_{Y} \leq \sum_{i=1}^{\infty} 2^{i-1} \varphi\left(0,-\frac{x}{(-2)^{i}}, \frac{x}{(-2)^{i}}\right)=\frac{1}{2} \widetilde{\varphi}(0,-x, x)
$$

for all $x \in X$.

Replacing $x, y, z$ by $\frac{x}{(-2)^{n}}, \frac{y}{(-2)^{n}}, \frac{z}{(-2)^{n}}$, respectively, and multiplying by $2^{n}$ in $(2.2)$, we get

$$
\begin{aligned}
& \left\|(-2)^{n} f\left(\frac{2 x}{(-2)^{n}}\right)+(-2)^{n} f\left(\frac{2 y}{(-2)^{n}}\right)+2 \cdot(-2)^{n} f\left(\frac{z}{(-2)^{n}}\right)\right\|_{Y} \\
\leq & \left\|2 \cdot(-2)^{n} f\left(\frac{x+y+z}{(-2)^{n}}\right)\right\|_{Y}+2^{n} \varphi\left(\frac{x}{(-2)^{n}}, \frac{y}{(-2)^{n}}, \frac{z}{(-2)^{n}}\right)
\end{aligned}
$$

for all $x, y, z \in X$. Since (2.5) gives that

$$
\lim _{n \rightarrow \infty} 2^{n} \varphi\left(\frac{x}{(-2)^{n}}, \frac{y}{(-2)^{n}}, \frac{z}{(-2)^{n}}\right)=0
$$

for all $x, y, z \in X$, if we let $n \rightarrow \infty$ in the above inequality, then we get

$$
\|L(2 x)+L(2 y)+2 L(z)\|_{Y} \leq\|2 L(x+y+z)\|_{Y}
$$

and so $L$ is additive by Lemma 2.1 .

The rest of the proof is the same as in the corresponding part of the proof of Theorem 2.2, as desired.

Lemma 2.4. Let $f: X \rightarrow Y$ be a mapping satisfying

$$
\|f(2 x)+\mu f(2 y)+2 f(z)\|_{Y} \leq\|2 f(x+\mu y+z)\|_{Y}
$$

for all $\mu \in \mathbb{T}^{1}$ and all $x, y, z \in X$. Then $f$ is $\mathbb{C}$-linear.

Proof. If we put $\mu=1$ in (2.7), then $f$ is additive by Lemma 2.1 .

Replacing $x, y, z$ by $\mu x,-x, 0$, respectively, we get $f(2 \mu x)+\mu f(-2 x)=0$ and so $f(\mu x)=\mu f(x)$ for all $\mu \in \mathbb{T}^{1}$ and all $x \in X$. Thus we have $f(\mu x+$ $\bar{\mu} x)=f(\mu x)+f(\bar{\mu} x)=\mu f(x)+\bar{\mu} f(x)$ for all $\mu \in \mathbb{T}^{1}$ and all $x \in X$, and so $f(t x)=t f(x)$ for any real number $t$ with $|t| \leq 1$ and all $x \in X$. 
On the other hand, since $f(2 x)=2 f(x)$, we get $f\left(2^{n} x\right)=2^{n} f(x)$ for all $n \in \mathbb{N}$. Since, for any real number $t$, there is a natural number $n$ with $|t| \leq 2^{n}$, we have

$$
f(t x)=f\left(2^{n} \cdot \frac{t}{2^{n}} x\right)=2^{n} f\left(\frac{t}{2^{n}} x\right)=2^{n} \cdot \frac{t}{2^{n}} f(x)=t f(x) .
$$

Now we consider any $\alpha \in \mathbb{C}$ with $\alpha=t+s i$ for some real numbers $t, s$. Since $f(i x)=i f(x)$ holds, we have

$$
f(\alpha x)=f(t x)+f(s i x)=t f(x)+s f(i x)=t f(x)+\operatorname{sif}(x)=\alpha f(x)
$$

and so $f$ is $\mathbb{C}$-linear, as desired.

Theorem 2.5. Let $f: X \rightarrow Y$ be a mapping with $f(0)=0$. If there exists a function $\varphi: X^{3} \rightarrow[0, \infty)$ satisfying $(2.3)$ and

$$
\|f(2 x)+\mu f(2 y)+2 f(z)\|_{Y} \leq\|2 f(x+\mu y+z)\|_{Y}+\varphi(x, y, z)
$$

for all $\mu \in \mathbb{T}^{1}$ and all $x, y, z \in X$, then there exists a unique $\mathbb{C}$-linear mapping $L: X \rightarrow Y$ satisfying (2.4).

Proof. If we put $\mu=1$ in (2.8), then by Theorem 2.2 there exists a unique additive mapping $L: X \rightarrow Y$ defined by

$$
L(x):=\lim _{n \rightarrow \infty} \frac{f\left((-2)^{n} x\right)}{(-2)^{n}}
$$

for all $x \in X$ which satisfies (2.4). By a similar method to the corresponding part of the proof of Theorem 2.2, $L$ satisfies

$$
\|L(2 x)+\mu L(2 y)+2 L(z)\|_{Y} \leq\|2 L(x+\mu y+z)\|_{Y}
$$

for all $\mu \in \mathbb{T}^{1}$ and all $x, y, z \in X$. Thus Lemma 2.4 gives that $L$ is $\mathbb{C}$-linear.

Corollary 2.6. Let $f: X \rightarrow Y$ be a mapping. If there exists a function $\varphi: X^{3} \rightarrow[0, \infty)$ satisfying $(2.5)$ and $(2.8)$, then there exists a unique $\mathbb{C}$-linear mapping $L: X \rightarrow Y$ satisfying (2.6).

Proof. If we put $\mu=1$ in (2.8), then by Corollary 2.3, there exists a unique additive mapping $L: X \rightarrow Y$ defined by

$$
L(x):=\lim _{n \rightarrow \infty}(-2)^{n} f\left(\frac{x}{(-2)^{n}}\right)
$$

for all $x \in X$ which satisfies (2.6).

The rest of the proof is the same as in the corresponding part of the proof of Theorem 2.5, as desired. 


\section{Stability of homomorphisms in proper $C Q^{*}$-algebras}

We investigate the Hyers-Ulam-Rassias stability of isomorphisms in proper $C Q^{*}$-algebras associated with the additive functional inequality. From now on, we suppose that $\left(A, A_{0}\right)$ and $\left(B, B_{0}\right)$ are proper $C Q^{*}$-algebras.

Theorem 3.1. Let $f: A \rightarrow B$ be a mapping with $f(0)=0$. If there exists a function $\varphi: A^{3} \rightarrow[0, \infty)$ satisfying

$$
\begin{aligned}
& \|f(2 x)+\mu f(2 y)+2 f(z)\|_{B} \leq\|2 f(x+\mu y+z)\|_{B}+\varphi(x, y, z), \\
& \widetilde{\varphi}(x, y, z):=\sum_{j=0}^{\infty} \frac{1}{2^{j}} \varphi\left((-2)^{j} x,(-2)^{j} y,(-2)^{j} z\right)<\infty
\end{aligned}
$$

for all $\mu \in \mathbb{T}^{1}$ and all $x, y, z \in A$, and, in addition, there exists a function $\phi: A^{2} \rightarrow[0, \infty)$ satisfying

$$
\begin{gathered}
\|f(x y)-f(x) f(y)\|_{B} \leq \phi(x, y), \\
\lim _{n \rightarrow \infty} \frac{1}{4^{n}} \phi\left((-2)^{n} x,(-2)^{n} y\right)=0
\end{gathered}
$$

for all $x, y \in A$ whenever the multiplication is defined, then there exists a unique proper $C Q^{*}$-algebra homomorphism $h: A \rightarrow B$ satisfying

$$
\|f(x)-h(x)\|_{B} \leq \frac{1}{2} \widetilde{\varphi}(0,-x, x)
$$

for all $x \in A$.

Proof. By Theorem 2.5, we have a unique $\mathbb{C}$-linear mapping $h: A \rightarrow B$ defined by

$$
h(x):=\lim _{n \rightarrow \infty} \frac{f\left((-2)^{n} x\right)}{(-2)^{n}}
$$

for all $x \in A$ which satisfies (3.5).

Now we show that $h(x y)=h(x) h(y)$ for all $x, y \in A$ whenever the multiplication is defined.

Replacing $x, y$ by $(-2)^{n} x,(-2)^{n} y$, respectively, and dividing by $4^{n}$ in (3.3), we get

$$
\left\|\frac{1}{4^{n}}\left[f\left((-2)^{n} x(-2)^{n} y\right)-f\left((-2)^{n} x\right) f\left((-2)^{n} y\right)\right]\right\|_{B} \leq \frac{1}{4^{n}} \phi\left((-2)^{n} x,(-2)^{n} y\right)
$$

for all $x, y \in A$ whenever the multiplication is defined. We have

$$
\begin{aligned}
\lim _{n \rightarrow \infty} \frac{1}{4^{n}} f\left((-2)^{n} x(-2)^{n} y\right) & =\lim _{n \rightarrow \infty} \frac{f\left((-2)^{2 n} x y\right)}{(-2)^{2 n}}=h(x y) \\
\lim _{n \rightarrow \infty} \frac{1}{4^{n}} f\left((-2)^{n} x\right) f\left((-2)^{n} y\right) & =\lim _{n \rightarrow \infty} \frac{f\left((-2)^{n} x\right)}{(-2)^{n}} \cdot \lim _{n \rightarrow \infty} \frac{f\left((-2)^{n} x\right)}{(-2)^{n}}=h(x) h(y)
\end{aligned}
$$

for all $x, y \in A$ whenever the multiplication is defined. If we let $n \rightarrow \infty$ in the above inequality, then (3.4) gives $h(x y)=h(x) h(y)$ for all $x, y \in A$ whenever the multiplication is defined. 
Corollary 3.2. Let $\theta, p$ be nonnegative real numbers with $p<1$ and let $f$ : $A \rightarrow B$ a mapping satisfying

$$
\begin{aligned}
\|f(2 x)+\mu f(2 y)+2 f(z)\|_{B} & \leq\|2 f(x+\mu y+z)\|_{B}+\theta\left(\|x\|_{A}^{p}+\|y\|_{A}^{p}+\|z\|_{A}^{p}\right), \\
\|f(x y)-f(x) f(y)\|_{B} & \leq \theta\left(\|x\|_{A}^{2 p}+\|y\|_{A}^{2 p}\right)
\end{aligned}
$$

for all $\mu \in \mathbb{T}^{1}$ and all $x, y, z \in A$ whenever the multiplication is defined. Then there exists a unique proper $C Q^{*}$-algebra homomorphism $h: A \rightarrow B$ satisfying

$$
\|f(x)-h(x)\|_{B} \leq \frac{2 \theta}{2-2^{p}}\|x\|_{A}^{p}
$$

for all $x \in A$.

Proof. Let $\varphi: A^{3} \rightarrow[0, \infty)$ be $\varphi(x, y, z)=\theta\left(\|x\|_{A}^{p}+\|y\|_{A}^{p}+\|z\|_{A}^{p}\right)$. When $p<1$, we get

$$
\begin{aligned}
\widetilde{\varphi}(x, y, z): & =\sum_{j=0}^{\infty} \frac{1}{2^{j}} \varphi\left((-2)^{j} x,(-2)^{j} y,(-2)^{j} z\right) \\
& =\sum_{j=0}^{\infty} \frac{2^{p j}}{2^{j}} \theta\left(\|x\|_{A}^{p}+\|y\|_{A}^{p}+\|z\|_{A}^{p}\right) \\
& =\frac{2 \theta}{2-2^{p}}\left(\|x\|_{A}^{p}+\|y\|_{A}^{p}+\|z\|_{A}^{p}\right) .
\end{aligned}
$$

In addition, let $\phi: A^{2} \rightarrow[0, \infty)$ be $\phi(x, y)=\theta\left(\|x\|_{A}^{2 p}+\|y\|_{A}^{2 p}\right)$. When $p<1$, we have

$$
\lim _{n \rightarrow \infty} \frac{1}{4^{n}} \phi\left((-2)^{n} x,(-2)^{n} y\right)=\lim _{n \rightarrow \infty} \frac{2^{2 p n}}{4^{n}} \theta\left(\|x\|_{A}^{2 p}+\|y\|_{A}^{2 p}\right)=0
$$

for all $x, y \in A$. By applying Theorem 3.1, there exists a unique proper $C Q^{*}$ algebra homomorphism $h: A \rightarrow B$ such that

$$
\|f(x)-h(x)\|_{B} \leq \frac{1}{2} \widetilde{\varphi}(0,-x, x)=\frac{2 \theta}{2-2^{p}}\|x\|_{A}^{p}
$$

for all $x \in A$.

Corollary 3.3. Let $\theta, p$ be nonnegative real numbers with $p<1$ and let $f$ : $A \rightarrow B$ a mapping satisfying

$$
\begin{aligned}
\|f(2 x)+\mu f(2 y)+2 f(z)\|_{B} & \leq\|2 f(x+\mu y+z)\|_{B}+\theta\left(\|x\|_{A}^{p}+\|y\|_{A}^{p}+\|z\|_{A}^{p}\right), \\
\|f(x y)-f(x) f(y)\|_{B} & \leq \theta \cdot\|x\|_{A}^{p} \cdot\|y\|_{A}^{p}
\end{aligned}
$$

for all $x, y, z \in A$ whenever the multiplication is defined. Then there exists a unique proper $C Q^{*}$-algebra homomorphism $h: A \rightarrow B$ satisfying

$$
\|f(x)-h(x)\|_{B} \leq \frac{2 \theta}{2-2^{p}}\|x\|_{A}^{p}
$$

for all $x \in A$. 
Proof. Let $\varphi: A^{3} \rightarrow[0, \infty)$ be $\varphi(x, y, z)=\theta\left(\|x\|_{A}^{p}+\|y\|_{A}^{p}+\|z\|_{A}^{p}\right)$ and let $\phi: A^{2} \rightarrow[0, \infty)$ be $\phi(x, y)=\theta \cdot\|x\|_{A}^{p} \cdot\|y\|_{A}^{p}$. When $p<1$, we have $\widetilde{\varphi}(x, y, z)<\infty$ and

$$
\lim _{n \rightarrow \infty} \frac{1}{4^{n}} \phi\left((-2)^{n} x,(-2)^{n} y\right)=\lim _{n \rightarrow \infty} \frac{2^{2 p n}}{4^{n}} \cdot \theta \cdot\|x\|_{A}^{p} \cdot\|y\|_{A}^{p}=0
$$

for all $x, y, z \in A$.

By applying Theorem 3.1, there exists a unique proper $C Q^{*}$-algebra homomorphism $h: A \rightarrow B$ such that

$$
\|f(x)-h(x)\|_{B} \leq \frac{1}{2} \widetilde{\varphi}(0,-x, x)=\frac{2 \theta}{2-2^{p}}\|x\|_{A}^{p}
$$

for all $x \in A$.

Theorem 3.4. Let $f: A \rightarrow B$ be a mapping. If there exists a function $\varphi: A^{3} \rightarrow[0, \infty)$ satisfying (3.1) and

$$
\widetilde{\varphi}(x, y, z):=\sum_{i=1}^{\infty} 2^{i} \varphi\left(\frac{x}{(-2)^{i}}, \frac{y}{(-2)^{i}}, \frac{z}{(-2)^{i}}\right)<\infty
$$

for all $x, y, z \in A$, and, in addition, there exists a function $\phi: A^{2} \rightarrow[0, \infty)$ satisfying (3.3) and

$$
\lim _{n \rightarrow \infty} 4^{n} \phi\left(\frac{x}{(-2)^{n}}, \frac{y}{(-2)^{n}}\right)=0
$$

for all $x, y \in A$ whenever the multiplication is defined, then there exists a unique proper $C Q^{*}$-algebra homomorphism $h: A \rightarrow B$ satisfying

$$
\|f(x)-h(x)\|_{B} \leq \frac{1}{2} \widetilde{\varphi}(0,-x, x)
$$

for all $x \in A$.

Proof. By Corollary 2.6, we have a unique $\mathbb{C}$-linear mapping $h: A \rightarrow B$ defined by

$$
h(x):=\lim _{n \rightarrow \infty}(-2)^{n} f\left(\frac{x}{(-2)^{n}}\right)
$$

for all $x \in A$ which satisfies (3.8).

Now replacing $x, y$ by $\frac{x}{(-2)^{n}}, \frac{y}{(-2)^{n}}$, respectively, and multiplying by $4^{n}$ in (3.3), we get

$$
\begin{aligned}
& \left\|4^{n}\left[f\left(\frac{x}{(-2)^{n}} \cdot \frac{y}{(-2)^{n}}\right)-f\left(\frac{x}{(-2)^{n}}\right) f\left(\frac{y}{(-2)^{n}}\right)\right]\right\|_{B} \\
\leq & 4^{n} \phi\left(\frac{x}{(-2)^{n}}, \frac{y}{(-2)^{n}}\right)
\end{aligned}
$$

for all $x, y \in A$ whenever the multiplication is defined. Since

$$
\lim _{n \rightarrow \infty} 4^{n} f\left(\frac{x}{(-2)^{n}} \cdot \frac{y}{(-2)^{n}}\right)=\lim _{n \rightarrow \infty}(-2)^{2 n} f\left(\frac{x y}{(-2)^{2 n}}\right)=h(x y)
$$




$$
\begin{aligned}
& \lim _{n \rightarrow \infty} 4^{n} f\left(\frac{x}{(-2)^{n}}\right) f\left(\frac{y}{(-2)^{n}}\right) \\
= & \lim _{n \rightarrow \infty}(-2)^{n} f\left(\frac{x}{(-2)^{n}}\right) \cdot \lim _{n \rightarrow \infty}(-2)^{n} f\left(\frac{y}{(-2)^{n}}\right)=h(x) h(y)
\end{aligned}
$$

for all $x, y \in A$ whenever the multiplication is defined. If we let $n \rightarrow \infty$ in the above inequality, then (3.7) gives $h(x y)=h(x) h(y)$ for all $x, y \in A$ whenever the multiplication is defined.

Corollary 3.5. Let $\theta, p$ be nonnegative real numbers with $p>1$ and let $f$ : $A \rightarrow B$ a mapping satisfying

$$
\begin{aligned}
\|f(2 x)+\mu f(2 y)+2 f(z)\|_{B} & \leq\|2 f(x+\mu y+z)\|_{B}+\theta\left(\|x\|_{A}^{p}+\|y\|_{A}^{p}+\|z\|_{A}^{p}\right), \\
\|f(x y)-f(x) f(y)\|_{B} & \leq \theta\left(\|x\|_{A}^{2 p}+\|y\|_{A}^{2 p}\right)
\end{aligned}
$$

for all $\mu \in \mathbb{T}^{1}$ and all $x, y, z \in A$ whenever the multiplication is defined. Then there exists a unique proper $C Q^{*}$-algebra homomorphism $h: A \rightarrow B$ satisfying

$$
\|f(x)-h(x)\|_{B} \leq \frac{2 \theta}{2^{p}-2}\|x\|_{A}^{p}
$$

for all $x \in A$.

Proof. Let $\varphi: A^{3} \rightarrow[0, \infty)$ be $\varphi(x, y, z)=\theta\left(\|x\|_{A}^{p}+\|y\|_{A}^{p}+\|z\|_{A}^{p}\right)$. When $p>1$, we get

$$
\begin{aligned}
\widetilde{\varphi}(x, y, z): & =\sum_{i=1}^{\infty} 2^{i} \varphi\left(\frac{x}{(-2)^{i}}, \frac{y}{(-2)^{i}}, \frac{z}{(-2)^{i}}\right) \\
& =\sum_{i=1}^{\infty} \frac{2^{i}}{2^{p i}} \theta\left(\|x\|_{A}^{p}+\|y\|_{A}^{p}+\|z\|_{A}^{p}\right) \\
& =\frac{2 \theta}{2^{p}-2}\left(\|x\|_{A}^{p}+\|y\|_{A}^{p}+\|z\|_{A}^{p}\right) .
\end{aligned}
$$

In addition, let $\phi: A^{2} \rightarrow[0, \infty)$ be $\phi(x, y)=\theta\left(\|x\|_{A}^{2 p}+\|y\|_{A}^{2 p}\right)$. When $p>1$, we get

$$
\lim _{n \rightarrow \infty} 4^{n} \phi\left(\frac{x}{(-2)^{n}}, \frac{y}{(-2)^{n}}\right)=\lim _{n \rightarrow \infty} \frac{4^{n}}{2^{2 p n}} \theta\left(\|x\|_{A}^{2 p}+\|y\|_{A}^{2 p}\right)=0
$$

for all $x, y \in A$.

By applying Theorem 3.4, there exists a unique proper $C Q^{*}$-algebra homomorphism $h: A \rightarrow B$ such that

$$
\|f(x)-h(x)\|_{B} \leq \frac{1}{2} \widetilde{\varphi}(0,-x, x)=\frac{2 \theta}{2^{p}-2}\|x\|_{A}^{p}
$$

for all $x \in A$. 
Corollary 3.6. Let $\theta, p$ be nonnegative real numbers with $p>1$ and let $f$ : $A \rightarrow B$ a mapping satisfying

$$
\begin{aligned}
\|f(2 x)+\mu f(2 y)+2 f(z)\|_{B} & \leq\|2 f(x+\mu y+z)\|_{B}+\theta\left(\|x\|_{A}^{p}+\|y\|_{A}^{p}+\|z\|_{A}^{p}\right), \\
\|f(x y)-f(x) f(y)\|_{B} & \leq \theta \cdot\|x\|_{A}^{p} \cdot\|y\|_{A}^{p}
\end{aligned}
$$

for all $\mu \in \mathbb{T}^{1}$ and all $x, y, z \in A$ whenever the multiplication is defined. Then there exists a unique proper $C Q^{*}$-algebra homomorphism $h: A \rightarrow B$ satisfying

$$
\|f(x)-h(x)\|_{B} \leq \frac{2 \theta}{2^{p}-2}\|x\|_{A}^{p}
$$

for all $x \in A$.

Proof. Let $\varphi: A^{3} \rightarrow[0, \infty)$ be $\varphi(x, y, z)=\theta\left(\|x\|_{A}^{p}+\|y\|_{A}^{p}+\|z\|_{A}^{p}\right)$ and let $\phi: A^{2} \rightarrow[0, \infty)$ be $\phi(x, y)=\theta \cdot\|x\|_{A}^{p} \cdot\|y\|_{A}^{p}$. When $p>1$, we have $\widetilde{\varphi}(x, y, z)<\infty$ and

$$
\lim _{n \rightarrow \infty} 4^{n} \phi\left(\frac{x}{(-2)^{n}}, \frac{y}{(-2)^{n}}\right)=\lim _{n \rightarrow \infty} \frac{4^{n}}{2^{2 p n}} \cdot \theta \cdot\|x\|_{A}^{p} \cdot\|y\|_{A}^{p}=0
$$

for all $x, y, z \in A$. By applying Theorem 3.4, there exists a unique proper $C Q^{*}$-algebra homomorphism $h: A \rightarrow B$ such that

$$
\|f(x)-h(x)\|_{B} \leq \frac{1}{2} \widetilde{\varphi}(0,-x, x)=\frac{2 \theta}{2^{p}-2}\|x\|_{A}^{p}
$$

for all $x \in A$.

\section{Stability of derivations on proper $C Q^{*}$-algebras}

We investigate the Hyers-Ulam-Rassias stability of derivations on proper $C Q^{*}$-algebras associated with the additive functional inequality.

Theorem 4.1. Let $f: A \rightarrow A$ be a mapping with $f(0)=0$. If there exists a function $\varphi: A^{3} \rightarrow[0, \infty)$ satisfying

$$
\begin{aligned}
& \|f(2 x)+\mu f(2 y)+2 f(z)\|_{A} \leq\|2 f(x+\mu y+z)\|_{A}+\varphi(x, y, z), \\
& \widetilde{\varphi}(x, y, z):=\sum_{j=0}^{\infty} \frac{1}{2^{j}} \varphi\left((-2)^{j} x,(-2)^{j} y,(-2)^{j} z\right)<\infty
\end{aligned}
$$

for all $\mu \in \mathbb{T}^{1}$ and all $x, y, z \in A$, and, in addition, there exists a function $\psi: A^{2} \rightarrow[0, \infty)$ satisfying

$$
\begin{gathered}
\|f(x y)-f(x) y-x f(y)\|_{A} \leq \psi(x, y), \\
\lim _{n \rightarrow \infty} \frac{1}{4^{n}} \psi\left((-2)^{n} x,(-2)^{n} y\right)=0
\end{gathered}
$$

for all $x, y \in A$ whenever the multiplication is defined, then there exists a unique derivation $\delta$ on $A$ satisfying

$$
\|f(x)-\delta(x)\|_{A} \leq \frac{1}{2} \widetilde{\varphi}(0,-x, x)
$$

for all $x \in A$. 
Proof. By Theorem 2.5, we have a unique $\mathbb{C}$-linear mapping $\delta: A \rightarrow A$ defined by

$$
\delta(x):=\lim _{n \rightarrow \infty} \frac{f\left((-2)^{n} x\right)}{(-2)^{n}}
$$

for all $x \in A$ which satisfies (4.5).

Now we show that $\delta(x y)=\delta(x) \delta(y)$ for all $x, y \in A$ whenever the multiplication is defined.

Replacing $x, y$ by $(-2)^{n} x,(-2)^{n} y$, respectively, and dividing by $4^{n}$ in (4.3), we get

$$
\begin{aligned}
& \left\|\frac{1}{4^{n}}\left[f\left((-2)^{n} x(-2)^{n} y\right)-f\left((-2)^{n} x\right)(-2)^{n} y-(-2)^{n} x f\left((-2)^{n} y\right)\right]\right\|_{A} \\
\leq & \frac{1}{4^{n}} \psi\left((-2)^{n} x,(-2)^{n} y\right)
\end{aligned}
$$

for all $x, y \in A$ whenever the multiplication is defined. We have

$$
\begin{aligned}
\lim _{n \rightarrow \infty} \frac{1}{4^{n}} f\left((-2)^{n} x(-2)^{n} y\right) & =\lim _{n \rightarrow \infty} \frac{f\left((-2)^{2 n} x y\right)}{(-2)^{2 n}}=\delta(x y), \\
\lim _{n \rightarrow \infty} \frac{1}{4^{n}} f\left((-2)^{n} x\right) \cdot(-2)^{n} y & =\lim _{n \rightarrow \infty} \frac{f\left((-2)^{n} x\right)}{(-2)^{n}} \cdot \frac{(-2)^{n} y}{(-2)^{n}}=\delta(x) y, \\
\lim _{n \rightarrow \infty} \frac{1}{4^{n}}(-2)^{n} x \cdot f\left((-2)^{n} y\right) & =\lim _{n \rightarrow \infty} \frac{(-2)^{n} x}{(-2)^{n}} \cdot \frac{(-2)^{n} y}{(-2)^{n}}=x \delta(y)
\end{aligned}
$$

for all $x, y \in A$ whenever the multiplication is defined. If we let $n \rightarrow \infty$ in the above inequality then (4.4) gives $\delta(x y)=\delta(x) y-x \delta(y)$ for all $x, y \in A$ whenever the multiplication is defined.

Corollary 4.2. Let $\theta, p$ be nonnegative real numbers with $p<1$ and let $f$ : $A \rightarrow A$ a mapping satisfying

$$
\begin{aligned}
\|f(2 x)+\mu f(2 y)+2 f(z)\|_{A} & \leq\|2 f(x+\mu y+z)\|_{A}+\theta\left(\|x\|_{A}^{p}+\|y\|_{A}^{p}+\|z\|_{A}^{p}\right), \\
\|f(x y)-f(x) y-x f(y)\|_{A} & \leq \theta\left(\|x\|_{A}^{2 p}+\|y\|_{A}^{2 p}\right)
\end{aligned}
$$

for all $\mu \in \mathbb{T}^{1}$ and for all $x, y, z \in A$ whenever the multiplication is defined. Then there exists a unique derivation $\delta$ on A satisfying

$$
\|f(x)-\delta(x)\|_{A} \leq \frac{2 \theta}{2-2^{p}}\|x\|_{A}^{p}
$$

for all $x \in A$.

Proof. Let $\varphi: A^{3} \rightarrow[0, \infty)$ be $\varphi(x, y, z)=\theta\left(\|x\|_{A}^{p}+\|y\|_{A}^{p}+\|z\|_{A}^{p}\right)$. When $p<1$, we get

$$
\widetilde{\varphi}(x, y, z):=\sum_{j=0}^{\infty} \frac{1}{2^{j}} \varphi\left((-2)^{j} x,(-2)^{j} y,(-2)^{j} z\right)
$$




$$
\begin{aligned}
& =\sum_{j=0}^{\infty} \frac{2^{p j}}{2^{j}} \theta\left(\|x\|_{A}^{p}+\|y\|_{A}^{p}+\|z\|_{A}^{p}\right) \\
& =\frac{2 \theta}{2-2^{p}}\left(\|x\|_{A}^{p}+\|y\|_{A}^{p}+\|z\|_{A}^{p}\right) .
\end{aligned}
$$

In addition, let $\psi: A^{2} \rightarrow[0, \infty)$ be $\psi(x, y)=\theta\left(\|x\|_{A}^{2 p}+\|y\|_{A}^{2 p}\right)$. When $p<1$, we have

$$
\lim _{n \rightarrow \infty} \frac{1}{4^{n}} \psi\left((-2)^{n} x,(-2)^{n} y\right)=\lim _{n \rightarrow \infty} \frac{2^{2 p n}}{4^{n}} \theta\left(\|x\|_{A}^{2 p}+\|y\|_{A}^{2 p}\right)=0
$$

for all $x, y \in A$. By applying Theorem 4.1, there exists a unique proper $C Q^{*}$ algebra homomorphism $h: A \rightarrow B$ such that

$$
\|f(x)-\delta(x)\|_{A} \leq \frac{1}{2} \widetilde{\varphi}(0,-x, x)=\frac{2 \theta}{2-2^{p}}\|x\|_{A}^{p}
$$

for all $x \in A$.

Corollary 4.3. Let $\theta, p$ be nonnegative real numbers with $p<1$ and let $f$ : $A \rightarrow B$ a mapping satisfying

$$
\begin{aligned}
&\|f(2 x)+\mu f(2 y)+2 f(z)\|_{A} \leq\|2 f(x+\mu y+z)\|_{A}+\theta\left(\|x\|_{A}^{p}+\|y\|_{A}^{p}+\|z\|_{A}^{p}\right), \\
&\|f(x y)-f(x) y-x f(y)\|_{A} \leq \theta \cdot\|x\|_{A}^{p} \cdot\|y\|_{A}^{p}
\end{aligned}
$$

for all $x, y, z \in A$ whenever the multiplication is defined. Then there exists a unique derivation $\delta$ on A satisfying

$$
\|f(x)-\delta(x)\|_{A} \leq \frac{2 \theta}{2-2^{p}}\|x\|_{A}^{p}
$$

for all $x \in A$.

Proof. Let $\varphi: A^{3} \rightarrow[0, \infty)$ be $\varphi(x, y, z)=\theta\left(\|x\|_{A}^{p}+\|y\|_{A}^{p}+\|z\|_{A}^{p}\right)$ and let $\psi$ : $A^{2} \rightarrow[0, \infty)$ be $\psi(x, y)=\theta \cdot\|x\|_{A}^{p} \cdot\|y\|_{A}^{p}$. When $p<1$, we have $\widetilde{\varphi}(x, y, z)<\infty$ and

$$
\lim _{n \rightarrow \infty} \frac{1}{4^{n}} \phi\left((-2)^{n} x,(-2)^{n} y\right)=\lim _{n \rightarrow \infty} \frac{2^{2 p n}}{4^{n}} \cdot \theta \cdot\|x\|_{A}^{p} \cdot\|y\|_{A}^{p}=0
$$

for all $x, y, z \in A$.

By applying Theorem 4.1, there exists a unique proper $C Q^{*}$-algebra homomorphism $\delta: A \rightarrow A$ such that

$$
\|f(x)-\delta(x)\|_{A} \leq \frac{1}{2} \widetilde{\varphi}(0,-x, x)=\frac{2 \theta}{2-2^{p}}\|x\|_{A}^{p}
$$

for all $x \in A$.

Theorem 4.4. Let $f: A \rightarrow A$ be a mapping. If there exists a function $\varphi$ : $A^{3} \rightarrow[0, \infty)$ satisfying $(4.1)$ and

$$
\widetilde{\varphi}(x, y, z):=\sum_{i=1}^{\infty} 2^{i} \varphi\left(\frac{x}{(-2)^{j}}, \frac{y}{(-2)^{j}}, \frac{z}{(-2)^{j}}\right)<\infty,
$$


and, in addition, there exists a function $\psi: A^{2} \rightarrow[0, \infty)$ satisfying (4.3) and

$$
\lim _{n \rightarrow \infty} 4^{n} \psi\left(\frac{x}{(-2)^{n}}, \frac{y}{(-2)^{n}}\right)=0
$$

for all $x, y \in A$, then there exists a unique derivation $\delta$ on A satisfying

$$
\|f(x)-\delta(x)\|_{A} \leq \frac{1}{2} \widetilde{\varphi}(0,-x, x)
$$

for all $x \in A$.

Proof. By Corollary 2.6, we have a unique $\mathbb{C}$-linear mapping $\delta: A \rightarrow A$ defined by

$$
\delta(x):=\lim _{n \rightarrow \infty}(-2)^{n} f\left(\frac{x}{(-2)^{n}}\right)
$$

for all $x \in A$ which satisfies (4.8).

Now replacing $x, y$ by $\frac{x}{(-2)^{n}}, \frac{y}{(-2)^{n}}$, respectively, and multiplying by $4^{n}$ in (4.3), we get

$$
\begin{aligned}
& \left\|4^{n}\left[f\left(\frac{x}{(-2)^{n}} \cdot \frac{y}{(-2)^{n}}\right)-f\left(\frac{x}{(-2)^{n}}\right) f\left(\frac{y}{(-2)^{n}}\right)\right]\right\|_{B} \\
\leq & 4^{n} \psi\left(\frac{x}{(-2)^{n}}, \frac{y}{(-2)^{n}}\right)
\end{aligned}
$$

for all $x, y \in A$ whenever the multiplication is defined. We have

$$
\begin{aligned}
& \lim _{n \rightarrow \infty} 4^{n} f\left(\frac{x}{(-2)^{n}} \cdot \frac{y}{(-2)^{n}}\right)=\lim _{n \rightarrow \infty}(-2)^{2 n} f\left(\frac{x y}{(-2)^{2 n}}\right)=\delta(x y), \\
& \lim _{n \rightarrow \infty} 4^{n} f\left(\frac{x}{(-2)^{n}}\right) \cdot \frac{y}{(-2)^{n}}=\lim _{n \rightarrow \infty}(-2)^{n} f\left(\frac{x}{(-2)^{n}}\right) \cdot \lim _{n \rightarrow \infty} \frac{(-2)^{n} y}{(-2)^{n}}=\delta(x) y, \\
& \lim _{n \rightarrow \infty} 4^{n} \frac{x}{(-2)^{n}} \cdot f\left(\frac{y}{(-2)^{n}}\right)=\lim _{n \rightarrow \infty} \frac{(-2)^{n} x}{(-2)^{n}} \cdot \lim _{n \rightarrow \infty}(-2)^{n} f\left(\frac{y}{(-2)^{n}}\right)=x \delta(y)
\end{aligned}
$$

for all $x, y \in A$ whenever the multiplication is defined. If we let $n \rightarrow \infty$ in the above inequality, then (4.7) gives $\delta(x y)=\delta(x) y-x \delta(y)$ for all $x, y \in A$ whenever the multiplication is defined.

Corollary 4.5. Let $\theta, p$ be nonnegative real numbers with $p>1$ and let $f$ : $A \rightarrow A$ a mapping satisfying

$$
\begin{aligned}
\|f(2 x)+\mu f(2 y)+2 f(z)\|_{B} & \leq\|2 f(x+\mu y+z)\|_{A}+\theta\left(\|x\|_{A}^{p}+\|y\|_{A}^{p}+\|z\|_{A}^{p}\right), \\
\|f(x y)-f(x) y-x f(y)\|_{A} & \leq \theta\left(\|x\|_{A}^{2 p}+\|y\|_{A}^{2 p}\right)
\end{aligned}
$$

for all $\mu \in \mathbb{T}^{1}$ and all $x, y, z \in A$ whenever the multiplication is defined. Then there exists a unique derivation $\delta$ on $A$ satisfying

$$
\|f(x)-\delta(x)\|_{A} \leq \frac{2 \theta}{2^{p}-2}\|x\|_{A}^{p}
$$

for all $x \in A$. 
Proof. Let $\varphi: A^{3} \rightarrow[0, \infty)$ be $\varphi(x, y, z)=\theta\left(\|x\|_{A}^{p}+\|y\|_{A}^{p}+\|z\|_{A}^{p}\right)$. When $p>1$, we get

$$
\begin{aligned}
\widetilde{\varphi}(x, y, z): & =\sum_{i=1}^{\infty} 2^{i} \varphi\left(\frac{x}{(-2)^{i}}, \frac{y}{(-2)^{i}}, \frac{z}{(-2)^{i}}\right) \\
& =\sum_{i=1}^{\infty} \frac{2^{i}}{2^{p i}} \theta\left(\|x\|_{A}^{p}+\|y\|_{A}^{p}+\|z\|_{A}^{p}\right) \\
& =\frac{2 \theta}{2^{p}-2}\left(\|x\|_{A}^{p}+\|y\|_{A}^{p}+\|z\|_{A}^{p}\right) .
\end{aligned}
$$

In addition, let $\psi: A^{2} \rightarrow[0, \infty)$ be $\phi(x, y)=\theta\left(\|x\|_{A}^{2 p}+\|y\|_{A}^{2 p}\right)$. When $p>1$, we get

$$
\lim _{n \rightarrow \infty} 4^{n} \psi\left(\frac{x}{(-2)^{n}}, \frac{y}{(-2)^{n}}\right)=\lim _{n \rightarrow \infty} \frac{4^{n}}{2^{2 p n}} \theta\left(\|x\|_{A}^{2 p}+\|y\|_{A}^{2 p}\right)=0
$$

for all $x, y \in A$.

By applying Theorem 4.4, there exists a unique proper $C Q^{*}$-algebra homomorphism $\delta: A \rightarrow A$ such that

$$
\|f(x)-\delta(x)\|_{A} \leq \frac{1}{2} \widetilde{\varphi}(0,-x, x)=\frac{2 \theta}{2^{p}-2}\|x\|_{A}^{p}
$$

for all $x \in A$.

Corollary 4.6. Let $\theta, p$ be nonnegative real numbers with $p>1$ and let $f$ : $A \rightarrow B$ a mapping satisfying

$$
\begin{aligned}
\|f(2 x)+\mu f(2 y)+2 f(z)\|_{A} & \leq\|2 f(x+\mu y+z)\|_{A}+\theta\left(\|x\|_{A}^{p}+\|y\|_{A}^{p}+\|z\|_{A}^{p}\right), \\
\|f(x y)-f(x) y-x f(y)\|_{A} & \leq \theta \cdot\|x\|_{A}^{p} \cdot\|y\|_{A}^{p}
\end{aligned}
$$

for all $\mu \in \mathbb{T}^{1}$ and all $x, y, z \in A$ whenever the multiplication is defined. Then there exists a unique derivation $\delta$ on $A$ satisfying

$$
\|f(x)-\delta(x)\|_{A} \leq \frac{2 \theta}{2^{p}-2}\|x\|_{A}^{p}
$$

for all $x \in A$.

Proof. Let $\varphi: A^{3} \rightarrow[0, \infty)$ be $\varphi(x, y, z)=\theta\left(\|x\|_{A}^{p}+\|y\|_{A}^{p}+\|z\|_{A}^{p}\right)$ and let $\psi$ : $A^{2} \rightarrow[0, \infty)$ be $\psi(x, y)=\theta \cdot\|x\|_{A}^{p} \cdot\|y\|_{A}^{p}$. When $p>1$, we have $\widetilde{\varphi}(x, y, z)<\infty$ and

$$
\lim _{n \rightarrow \infty} 4^{n} \phi\left(\frac{x}{(-2)^{n}}, \frac{y}{(-2)^{n}}\right)=\lim _{n \rightarrow \infty} \frac{4^{n}}{2^{2 p n}} \cdot \theta \cdot\|x\|_{A}^{p} \cdot\|y\|_{A}^{p}=0
$$

for all $x, y, z \in A$. By applying Theorem 3.4, there exists a unique proper $C Q^{*}$-algebra homomorphism $\delta: A \rightarrow A$ such that

$$
\|f(x)-\delta(x)\|_{A} \leq \frac{1}{2} \widetilde{\varphi}(0,-x, x)=\frac{2 \theta}{2^{p}-2}\|x\|_{A}^{p}
$$

for all $x \in A$. 
Acknowledgements. The first author and the third author were supported by Basic Science Research Program through the National Research Foundation of Korea funded by the Ministry of Education, Science and Technology (NRF2010-0009232) and (NRF-2010-0021792), respectively.

\section{References}

[1] J. P. Antoine, A. Inoue, and C. Trapani, $O^{*}$-dynamical systems and $*$-derivations of unbounded operator algebras, Math. Nachr. 204 (1999), 5-28.

[2] _ Partial *-Algebras and Their Operator Realizations, Kluwer, Dordrecht, 2002.

[3] F. Bagarello, Applications of topological *-algebras of unbounded operators, J. Math. Phys. 39 (1998), no. 11, 6091-6105.

[4] F. Bagarello, A. Inoue, and C. Trapani, Some classes of topological quasi *-algebras, Proc. Amer. Math. Soc. 129 (2001), no. 10, 2973-2980.

[5] , Derivations of quasi *-algebras, Int. J. Math. Math. Sci. 2004 (2004), no. 21-24, 1077-1096.

[6] _ Exponentiating derivations of quasi *-algebras: possible approaches and applications, Int. J. Math. Math. Sci. 2005 (2005), no. 17, 2805-2820.

[7] F. Bagarello and C. Trapani, States and representations of $C Q^{*}$-algebras, Ann. Inst. H. Poincaré Phys. Théor. 61 (1994), no. 1, 103-133.

[8],$C Q^{*}$-algebras: structure properties, Publ. Res. Inst. Math. Sci. 32 (1996), no. $1,85-116$.

[9] S. Czerwik, Functional Equations and Inequalities in Several Variables, World Scientific Publishing Company, New Jersey, London, Singapore and Hong Kong, 2002.

[10] Stability of Functional Equations of Ulam-Hyers-Rassias Type, Hadronic Press, Palm Harbor, Florida, 2003.

[11] Z. Gajda, On stability of additive mappings, Int. J. Math. Math. Sci. 14 (1991), no. 3, 431-434.

[12] P. Găvruta, A generalization of the Hyers-Ulam-Rassias stability of approximately additive mappings, J. Math. Anal. Appl. 184 (1994), no. 3, 431-436.

[13] D. H. Hyers, On the stability of the linear functional equation, Proc. Nat. Acad. Sci. U.S.A. 27 (1941), 222-224.

[14] D. H. Hyers, G. Isac, and Th. M. Rassias, Stability of Functional Equations in Several Variables, Birkhäuser, Basel, 1998.

[15] D. H. Hyers and Th. M. Rassias, Approximate homomorphisms, Aequationes Math. 44 (1992), no. 2-3, 125-153.

[16] C. Park, Lie *-homomorphisms between Lie $C^{*}$-algebras and Lie *-derivations on Lie $C^{*}$-algebras, J. Math. Anal. Appl. 293 (2004), no. 2, 419-434.

[17] $\quad$ Homomorphisms between Lie $J C^{*}$-algebras and Cauchy-Rassias stability of Lie $J C^{*}$-algebra derivations, J. Lie Theory 15 (2005), no. 2, 393-414.

[18] _ Isomorphisms between unital $C^{*}$-algebras, J. Math. Anal. Appl. 307 (2005), no. $2,753-762$

[19] _ Approximate homomorphisms on JB*-triples, J. Math. Anal. Appl. 306 (2005), no. $1,375-381$

[20] _ Isomorphisms between $C^{*}$-ternary algebras, J. Math. Phys. 47 (2006), no. 10, $103512,12 \mathrm{pp}$.

[21] _ Hyers-Ulam-Rassias stability of a generalized Apollonius-Jensen type additive mapping and isomorphisms between $C^{*}$-algebras, Math. Nachr. 281 (2008), no. 3, 402411.

[22] J. M. Rassias, On approximation of approximately linear mappings by linear mappings, J. Funct. Anal. 46 (1982), no. 1, 126-130. 
[23] _ On approximation of approximately linear mappings by linear mappings, Bull. Sci. Math. (2) 108 (1984), no. 4, 445-446.

[24] _ Solution of a problem of Ulam, J. Approx. Theory 57 (1989), no. 3, 268-273.

[25] On the stability of the linear mapping in Banach spaces, Proc. Amer. Math. Soc. 72 (1978), no. 2, 297-300.

[26] Problem 16; 2, Report of the $27^{\text {th }}$ International Symp. on Functional Equations, Aequationes Math. 39 (1990), 292-293; 309.

[27] _ The problem of S. M. Ulam for approximately multiplicative mappings, J. Math. Anal. Appl. 246 (2000), no. 2, 352-378.

[28] - On the stability of functional equations in Banach spaces, J. Math. Anal. Appl. 251 (2000), no. 1, 264-284.

[29] - On the stability of functional equations and a problem of Ulam, Acta Appl. Math. 62 (2000), no. 1, 23-130.

[30] - Functional Equations, Inequalities and Applications, Kluwer Academic Publishers, Dordrecht, Boston and London, 2003.

[31] Th. M. Rassias and P. Šemrl, On the Hyers-Ulam stability of linear mappings, J. Math. Anal. Appl. 173 (1993), no. 2, 325-338.

[32] F. Skof, Proprietà localie approssimazione di operatori, Rend. Sem. Mat. Fis. Milano 53 (1983), 113-129.

[33] C. Trapani, Quasi-*-algebras of operators and their applications, Rev. Math. Phys. 7 (1995), no. 8, 1303-1332.

[34] _ Some seminorms on quasi-*-algebras, Studia Math. 158 (2003), no. 2, 99-115.

[35] _ Bounded elements and spectrum in Banach quasi *-algebras, Studia Math. 172 (2006), no. 3, 249-273.

[36] S. M. Ulam, Problems in Modern Mathematics, Wiley, New York, 1960.

Jung RyE LEE

Department of Mathematics

DAEJIN UNIVERSITY

KYEONGGI 487-711, KoreA

E-mail address: jrlee@daejin.ac.kr

Choonkil Park

Department of Mathematics

Research Institute for Natural Sciences

HANYANG UNIVERSITY

SEOUl 133-791, KoreA

E-mail address: baak@hanyang.ac.kr

DONG Yun SHIN

Department of Mathematics

UNIVERSITy OF SEOUL

SEOUl 130-743, KoreA

E-mail address: dyshin@uos.ac.kr 